\title{
Transgenic cardiac-targeted overexpression of human thymidylate kinase
}

\author{
James J Kohler ${ }^{1, *}$, Seyed H Hosseini ${ }^{1, *}$, Ioan Cucoranu ${ }^{1}$, Olga Zhelyabovska ${ }^{2}$, Elgin Green ${ }^{1}$, Kristopher Ivey ${ }^{1}$, \\ Allison Abuin ${ }^{1}$, Earl Fields ${ }^{1}$, Amy Hoying ${ }^{1}$, Rodney Russ ${ }^{1}$, Robert Santoianni ${ }^{1}$, C Michael Raper ${ }^{1}$, Qinglin Yang ${ }^{2}$, \\ Arnon Lavie ${ }^{3}$ and William Lewis ${ }^{1}$
}

Thymidylate kinase (TMPK) is a nucleoside monophosphate kinase that catalyzes phosphorylation of thymidine monophosphate to thymidine diphosphate. TMPK also mediates phosphorylation of monophosphates of thymidine nucleoside analog (NA) prodrugs on the pathway to their active triphosphate antiviral or antitumor moieties. Novel transgenic mice (TG) expressing human (h) TMPK were genetically engineered using the $\alpha$-myosin heavy chain promoter to drive its cardiac-targeted overexpression. In ' 2 by 2' protocols, TMPK TGs and wild-type (WT) littermates were treated with the NA zidovudine (a deoxythymidine analog, 3'-azido-3'deoxythymidine (AZT)) or vehicle for 35 days. Alternatively, TGs and WTs were treated with a deoxycytidine NA (racivir, RCV) or vehicle. Changes in mitochondrial DNA (mtDNA) abundance and mitochondrial ultrastructure were defined quantitatively by real-time PCR and transmission electron microscopy, respectively. Cardiac performance was determined echocardiographically. Results showed TMPK TGs treated with either AZT or RCV exhibited decreased cardiac mtDNA abundance. Cardiac ultrastructural changes were seen only with AZT. AZT-treated TGs exhibited increased left ventricle (LV) mass. In contrast, LV mass in RCV-treated TGs and WTs remained unchanged. In all cohorts, LV end-diastolic dimension remained unchanged. This novel cardiac-targeted overexpression of hTMPK helps define the role of TMPK in mitochondrial toxicity of antiretrovirals.

Laboratory Investigation (2010) 90, 383-390; doi:10.1038/labinvest.2009.146; published online 11 January 2010

KEYWORDS: TMPK; mitochondrial toxicity; antiretrovirals; cardiac hypertrophy

Mitochondria are unique cellular organelles with their own DNA (mitochondrial DNA, mtDNA), replication machinery (polymerase gamma, pol $\gamma$ ), ${ }^{1}$ and mitochondrial deoxynucleotide triphosphate (dNTP) pools essential for the synthesis of mtDNA. ${ }^{2}$ mtDNA synthesis occurs throughout the life of the cell, independent of nuclear DNA (nDNA) synthesis and is essential for mitochondrial energy production. Cardiomyocytes (terminally differentiated, nonreplicating cells) require constant high-energy phosphates to sustain muscular contractions. Disrupted mtDNA replication can lead to left ventricle (LV) hypertrophy, cardiomegaly, and organ dysfunction.

The importance of maintaining the dNTP precursor pools is underscored by human genetic diseases in which mutations in enzymes for dNTP synthesis adversely affect the rate and/ or fidelity of mtDNA replication. ${ }^{3-5}$ Mitochondrial deoxythymidine triphosphate (dTTP) may be generated by two separate pathways in mammalian cells. De novo synthesis is accomplished from ribonucleotides in the cytosol and is followed by import into the mitochondria. ${ }^{6}$ Alternatively, deoxythymidine (dThd) is imported into the mitochondria and three sequential kinase-mediated phosphorylations generate dTTP. Cycling cells largely depend on the first pathway (de novo synthesis), whereas resting cells use the alternative pathway mediated by three mitochondrial kinases. ${ }^{7}$ Intramitochondrially, the first phosphorylation is mediated by the mitochondrial thymidine kinase (TK2), ${ }^{8}$ which we have studied earlier. ${ }^{9,10}$ The second phosphorylation is mediated by the cytosolic thymidylate kinase (TMPK). ${ }^{11}$ In this study, we targeted cytoplasmic

\footnotetext{
${ }^{1}$ Department of Pathology, Emory University School of Medicine, Atlanta, GA, USA; ${ }^{2}$ Department of Nutrition Sciences, University of Alabama at Birmingham, Birmingham, AL, USA and ${ }^{3}$ Department of Biochemistry and Molecular Genetics, University of Illinois at Chicago, Chicago, IL, USA

Correspondence: Dr JJ Kohler, PhD, Department of Pathology, Emory University School of Medicine, 7126 Woodruff Memorial Building, 101 Woodruff Circle, Atlanta, GA 30322, USA.

${ }^{* *}$ Co-first authors.
}

Received 28 August 2009; revised 29 October 2009; accepted 29 October 2009 
overexpression of TMPK. We hypothesized that in these TMPK transgenic mice (TGs), thymidine diphosphate (TDP) precursors in the cytoplasm would accumulate as the myocytes are quiescent. These precursors ultimately would be delivered into the mitochondria through specific nucleotide transporters (eg SLC25 ${ }^{12}$ ) where they could potentially impact mtDNA replication.

Nucleoside analogs (NA) are well-recognized anticancer and antiviral agents. ${ }^{13}$ The thymidine NA zidovudine ( $3^{\prime}$-azido-3'deoxythymidine, AZT) is a bona fide treatment for HIV/AIDS. NAs are 'prodrugs' whose therapeutic activity relates to conversion to the active triphosphated $N$ (eg, AZTTP). ${ }^{11,14,15}$ Like their native counterparts, NAs are phosphorylated serially into TPs mediated by the same kinases (eg, TK2 and TMPK). For AZT, the rate-limiting step is the conversion of AZTMP to AZTDP by TMPK.

As treatment with AZT is associated with mitochondrial toxicity to tissues, a novel in vivo model using overexpression of TMPK was engineered to delineate the role of TMPK in phosphorylation of native nucleosides and NAs. Using ' 2 by 2 ' protocols, TGs and wild types (WTs) were treated by daily gavage with each nucleoside analog solubolized in appropriate vehicles (AZT in carboxymethylcellulose (CMC) or racivir (RCV) in saline), and compared to respective vehicle controls. Experiments here characterize a novel cardiac-targeted TMPK TG and highlight the role of TMPK in mtDNA replication, cardiac function, and how TMPK determines toxicity of NAs.

\section{MATERIALS AND METHODS \\ Generation of Alpha-Myosin Heavy Chain Promoter- Driven hTMPK TG Mice}

Established methods ${ }^{16}$ were used as described earlier. ${ }^{17}$ They applied to the hTMPK cDNA construct. ${ }^{18}$ The TMPK gene included the R200A mutation. This mutation was initially done to facilitate structural studies on TMPK, and it does not affect the kinetic behavior of the enzyme. ${ }^{19}$ Three transgenic lines $(\mathrm{A}, \mathrm{B}, \mathrm{C})$ were established for the targeted overexpression of hTMPK. FVB/n (JAX stock, Jackson Lab, Bar Harbor, ME, USA) was the inbred background. Animals bred true for five generations (to ensure germline transgenesis) before beginning experimental studies. No gross phenotype was recognized in TGs or WT littermates. No changes in behavior, growth, maturation, breeding behavior, or Mendelian distribution of TG occurred.

\section{TG Gene Copy Analysis}

To determine the relative copy number in each line, the level of hTMPK was analyzed semi-quantitatively from murine tail DNA extracts using real-time PCR and Light Cycler TaqMan Master kit. Target genes were amplified using specific primers for hTMPK (forward TMPK/LC: $5^{\prime}$-ATGAGAACGGGGCTTTCC- ${ }^{\prime}$, reverse TMPK/LCR: $5^{\prime}$-TTTGGAAGCATCCACCATCT-3' ${ }^{\prime}$, and Universal Probe Library probe \#31; Roche Diagnostics Corp., Indianapolis,
IN, USA) and a 'housekeeping' gene, GAPDH (forward: 5'GATGCTACAAGCAGGCCTTT- $3^{\prime}$, reverse: 5'-GCAGAAAGC AAGGGCAAA-3', and Universal ProbeLibrary probe \#4; Roche Diagnostics Corp.). DNA amplification was performed using LightCycler 480 (Roche Diagnostics Corp.) on individual tissues extracted from at least five to seven mice within each line. Relative copy number dosage was normalized to endogenous murine TMPK (single copy gene) from WT.

Further verification/selection for the hTMPK was accomplished using PCR amplification of tail DNA using two specific primers that identify part of the $\alpha$-MHC promoter in tandem with the hTMPK gene, thereby avoiding amplification of the endogenous murine TMPK (TMPK foward 5'-CACATAGAAGCCTAGCCCACA- $3^{\prime}$ and TMPK reverse 5'-TATAGTCGACTCACTTCCATAGCTCCCACAGCGG-3').

\section{Antiretroviral Treatment Protocols}

All procedures complied with Emory Institutional Animal Care and Use Committee and NIH guidelines. WT and TG littermates (both genders) were age-matched (8-12 weeks) at the start of treatment protocols. Food and water were provided ad libitum in a $12 \mathrm{~h}$ light/dark, humidity and temperature controlled environment at Emory. Antiretroviral drugs were from the manufacturers (compliments of Emory Center for AIDS Research Pharmacology Core). Dosing was by daily gavage (morning). Treatment regimens resembled those used in human therapy and included AZT $(0.22 \mathrm{mg} /$ day; $0.25 \mathrm{ml}$ in $1 \% \mathrm{CMC}), \mathrm{RCV}(1.025 \mathrm{mg}$ /day in saline), or respective vehicles alone. At day 35 , animal weights and echocardiographic measurements were made ${ }^{20}$ and animals were terminated. This termination date was chosen experimentally based on our previous data that show measurable changes in cardiac function occur within 35 days of treatment with AZT. ${ }^{9,10,21-23}$ Heart samples were retrieved rapidly and frozen $\left(-80^{\circ} \mathrm{C}\right.$ storage) for subsequent DNA extraction and analysis, or were fixed for histology (10\% neutral buffered formalin; Fisher Scientific, Pittsburgh, PA, USA) and electron microscopy (EM).

\section{Echocardiography of TG Mice}

LV mass and LV end-diastolic dimension (LVEDD) were determined as described earlier using echocardiography (ECHO) in age- and gender-matched (littermate) WT and TGs. ${ }^{23}$ Briefly, LV mass was calculated from LV wall thickness and dimension measurements using an established formula.

\section{mtDNA and nDNA Quantification in Heart Tissue Using Real-Time PCR}

Methods used were based on modifications of those used by others ${ }^{24}$ and detailed by us. ${ }^{9,25}$ Amplification was performed in a LightCycler 480 (Roche Diagnostics). Efficiency curves corresponding to mitochondrial and nDNA were used to determine the ratio of mtDNA to nDNA in each sample. For mtDNA abundance, the resultant values were expressed as 
mean \pm standard error (s.e.m.), normalized to vehicle-treated WT mean (set at 1.0). A value of $P<0.05$ was considered statistically significant.

\section{Fine Structure Pathological Evaluations of Mitochondrial Changes with Transmission EM of Mitochondrial Damage in hTMPK TGs}

Mitochondrial ultrastructure was evaluated using transmission EM to support data from ECHO and mtDNA abundance using methods that resemble those used regularly in the laboratory. ${ }^{26}$ Each EM photograph was reviewed independently by two investigators for the presence of structurally abnormal mitochondria, increased numbers of mitochondrial profiles per field, intramitochondrial lamellar bodies, abnormal cristae density, cristae reduplication, mitochondrial swelling, and intramitochondrial paracrystals as performed by others. ${ }^{27}$

\section{Histological Analysis}

Heart samples were processed, sectioned $(6 \mu \mathrm{m})$, stained with hematoxylin and eosin (H\&E), and examined microscopically as done by us earlier. ${ }^{28}$ Images were stored electronically to compare histopathological features.

\section{Experimental Analysis and Statistics}

Values for LV mass, LVEDD, and mtDNA were compared in WT, TGs, and NA-treated cohorts using ANOVA in GraphPad Prism 4 (GraphPad, San Diego, CA, USA). Post hoc testing used Newman-Keul's and unpaired $t$-test. A value of
$P<0.05$ (determined by Student's unpaired $t$-test) was considered statistically significant.

\section{RESULTS \\ hTMPK Cardiac-Targeted Transgenic Design}

The mutant human TMPK clone as pGEX-RB plasmid was used. ${ }^{18}$ hTMPK was directionally inserted into a plasmid construct containing the alpha-myosin heavy chain promoter $(\alpha-\mathrm{MyHC})^{16}$ using the nucleoside restriction sites SalI and HindIII (Figure 1a). This $\alpha$-MyHC-hTMPK construct targeted overexpression of hTMPK in cardiac myocytes, based on primary activation of the $\alpha$-MyHC promoter activity exclusively in cardiomyocytes. Primers designed to amplify the region overlapping $\alpha$-MyHC promoter and $h T M P K$ gene $(\sim 750 \mathrm{bp})$ provided a way to identify the transgene unambiguously while preventing amplification of the endogenous murine TMPK gene. Dot blot imaging was obtained routinely on DNA isolated from tail clippings for F1-F5 progeny. Signal for positive TGs was compared to WT progeny, TMPK positive (plasmid DNA, dark dots) and negative controls (no plasmid, white dots) as indicated (Figure 1b). Semi-quantitation of hTMPK copy number was accomplished from the signals on the dotblots (eg, row A: positions 3-4, 6-7, and 9, high copy) versus other positive dot blots (eg, row B: positions 1-2, 5-8, medium copy). Alternatively, all subsequent generations of progeny were routinely identified using PCR amplification with positive TGs identified by the production of a $\sim 750 \mathrm{bp}$ band (Figure 1c).
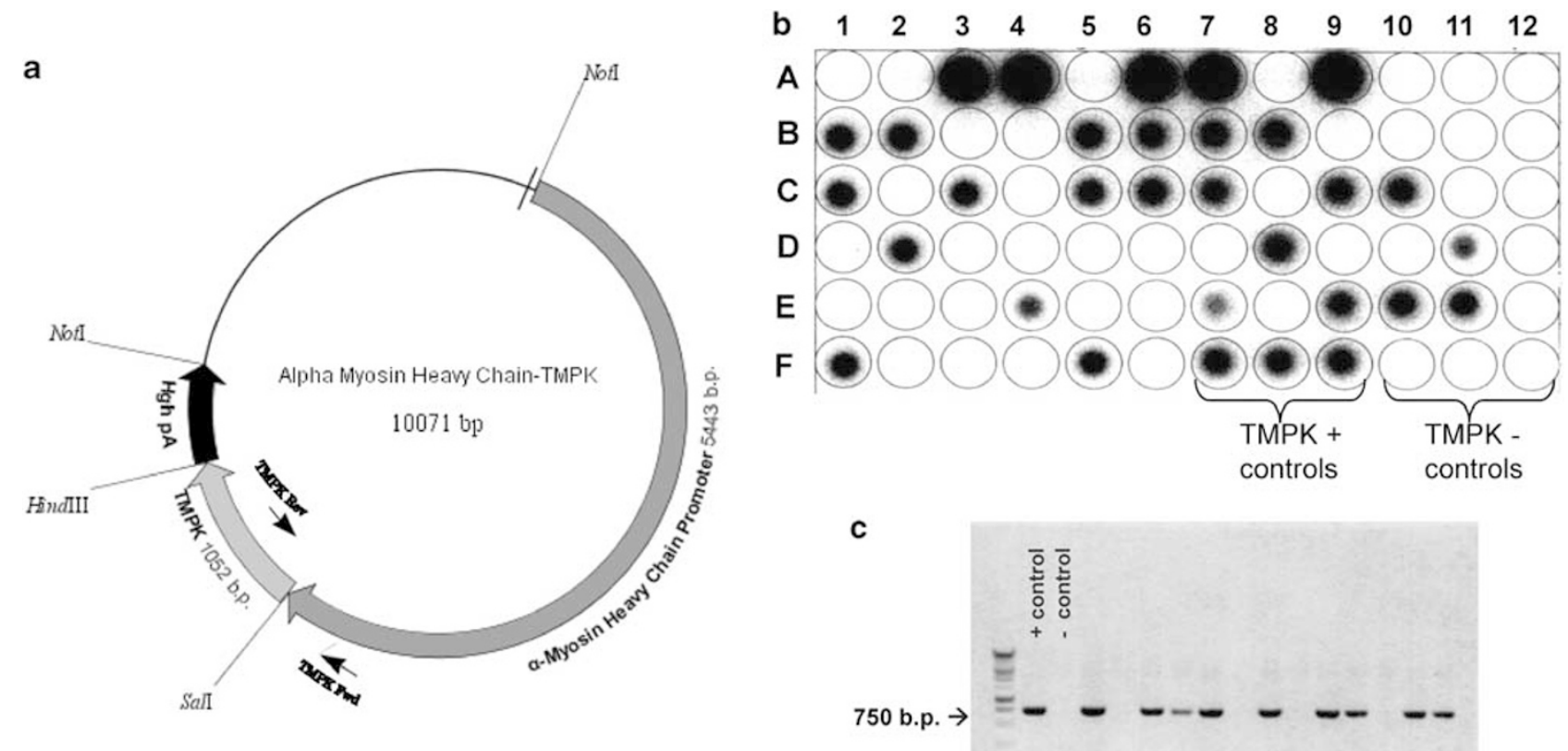

Figure 1 Generation and verification of TMPK transgenesis. (a) Molecular map summarizes $\alpha$-MyHC-TMPK construct with restriction sites and primerbinding sites for positive identification of transgenes. Representative dot blot (b) and agarose gel image of PCR amplification products (c) show selection of positive TGs from tail DNA of individual progeny from TG line(s) derived. 


\section{Murine TG Characteristics}

Cardiac-targeted transgenic overexpression of hTMPK was accomplished in three lines (operationally labeled as line A, $\mathrm{B}$, and $\mathrm{C}$ ). Animals bred true for five generations. In general, no gross phenotypic changes occurred in TGs or FVB/n WTs. No changes in behavior, growth, maturation, breeding behavior, or Mendelian distribution of TGs were found in any of the three TG lines.

The relative hTMPK gene copy number in the lines was semi-quantitatively determined (Table 1). All demonstrated multiple copy numbers of hTMPK. Lines A and B exhibited $\sim 5$-fold in excess of $\mathrm{WT}(\mathrm{FVB} / \mathrm{n}$, single murine copy) whereas TG C line exhibited 25 -fold excess. Line $C$ was selected for these initial pharmacological studies based on its high copy number. It was reasoned that functional impact of TMPK TG on mtDNA biogenesis and cardiac function with

Table 1 hTMPK TG gene copy number

\begin{tabular}{lcl}
\hline Line & TMPK gene dosage $^{\text {a }}$ & Copy number \\
\hline A & 5 & Medium \\
B & 5 & Medium \\
C & 25 & High
\end{tabular}

$\mathrm{FVB} / \mathrm{n}$ WT

1

Single copy

a Relative TMPK copy number normalized to FVB/n WT (single copy gene). or without an NRTI (NA) treatment would be most notable in a high copy number line.

\section{mtDNA Abundance}

Age-matched cohorts of TGs and WTs were treated with AZT (CMC vehicle), RCV (saline vehicle), or the respective vehicle control for 35 days. At study termination, mice were killed and extracts of total DNA were isolated from cardiac tissues from individual mice for each cohort. Both mtDNA and nDNA steady-state levels were determined using real-time PCR and resultant mtDNA/nDNA ratios (mtDNA abundance) were calculated and normalized as mean \pm s.e.m. Vehicle-treated TMPK TGs exhibited a small decrease (although not statistically significant, $P>0.05$ ) in mtDNA abundance compared to vehicle-treated WTs (Figure 2a and b).

Likewise, AZT had only a moderate effect on mtDNA abundance in WTs (Figure 2a). This effect can be attributed to the endogenous activity of mouse TMPK. Mouse TMPK is more active than its human counterpart with AZTMP. ${ }^{29}$ Therefore, in mice more AZT-triphosphate is generated relative to the condition in human cells. It is this activation of AZT by endogenous mouse TMPK that moderately decreases the mtDNA-abundance. In the case of RCV treated WT animals, no decrease in mtDNA is observed (Figure $2 b$ ).

In contrast, AZT-treated TGs exhibited a significant decrease in cardiac mtDNA abundance $(P<0.001$ and $<0.01$, respectively) (Figure 2a), suggesting disruption of mitochondrial biogenesis. With RCV treatment, TMPK TGs exhibited a significant decrease in mtDNA abundance only when compared to WTs (Figure 2b).

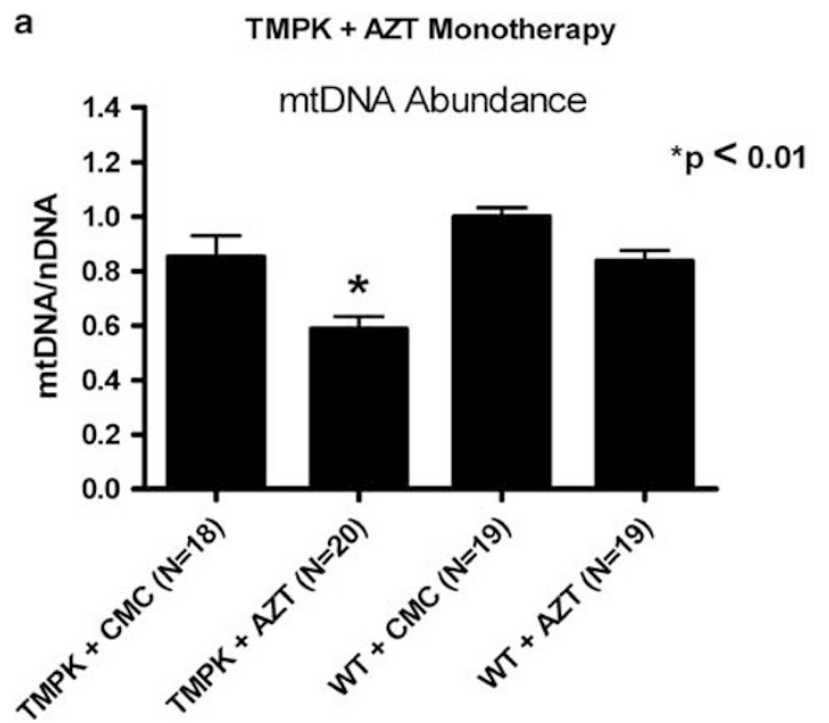

b

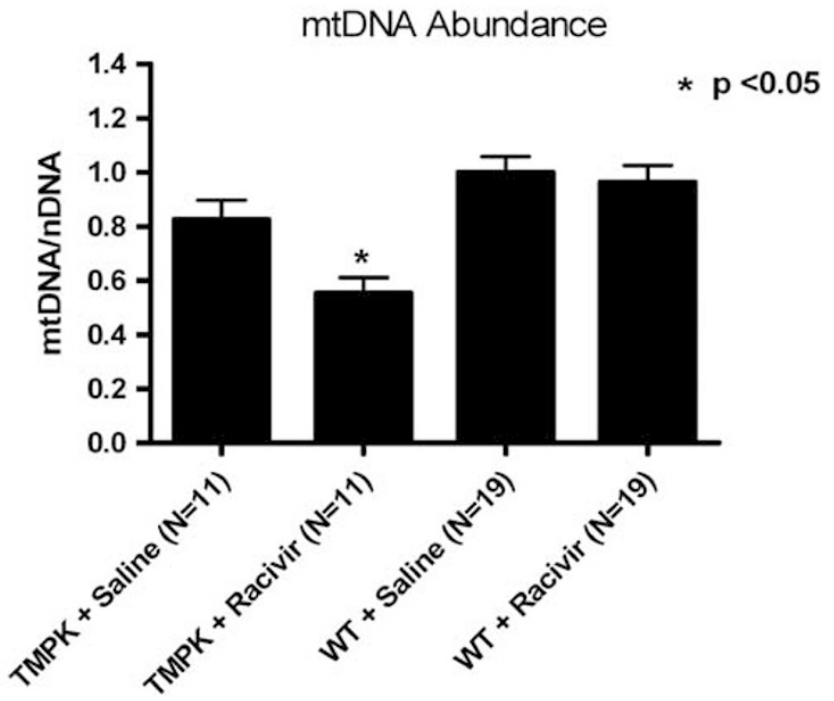

Figure 2 Cardiac mtDNA abundance in TMPK TGs and WTs after NRTI treatment. Total DNA was extracted from cardiac tissues isolated from TMPK TG and WT cohorts treated with zidovudine (AZT in CMC vehicle), racivir (RCV in saline vehicle), or vehicle alone for 35 days. mtDNA abundance was assessed using a ratio of mtDNA/nDNA as determined by real-time PCR amplification. Cardiac mtDNA abundance was significantly decreased in TMPK TGs treated with AZT (a) or RCV (b) compared to WT littermates. 


\section{Ultrastructural (EM) Features of the Mitochondria in TG Hearts}

EM profiles of cardiac myocytes from hTMPK TGs and WTs treated with AZT, RCV, or respective vehicle were assessed from formalin-fixed cardiac tissues. Ultrastructural features in mitochondrial structure from vehicle-treated TGs (both $\mathrm{CMC}$ and saline) were essentially unchanged compared to WT littermates (Figure 3a and b). Likewise, mitochondria from AZT- or RCV-treated WTs appeared unremarkable when compared to their vehicle-treated littermates. Mitochondria of cardiac myocytes from AZT-treated TGs, however, exhibited disruption of sarcomeres and tubules, mitochondrial swelling, and decreased cristae density compared to all other cohorts (Figure 3a, lower right panel). These findings correlate with decreased mtDNA abundance in cardiac samples from AZT-treated TGs and together suggest disruption of mitochondrial biogenesis. In contrast, mitochondria from RCV-treated TGs exhibited essentially no ultrastructural changes (Figure 3b, lower right panel) and appeared identical to WT littermates.

\section{ECHO Evalution of LV Mass in Hearts of TMPK TGs with and without Antiretroviral Therapy}

Both LV mass and LVEDD offer direct assessments of cardiac function and mass. An increase in either parameter is characteristic of cardiac dysfunction and suggestive of cardiac hypertrophy (increased LV mass) and/or dilation (increased LVEDD). ECHO data were recorded at termination of studies for individual mice from each cohort of hTMPK TGs and WTs with and without AZT or RCV treatments (35 days). LV mass for vehicle-treated (CMC or saline) TGs remained unchanged compared to WT littermates (Figure 4a and b, $P>0.05)$. AZT treatment led to increased LV mass in both WTs and TGs compared to vehicle (CMC)-treated WT (Figure $4 \mathrm{a}, P<0.05$ ), suggesting LV hypertrophy. No significant change in LV mass was found after RCV treatment compared to vehicle (saline)-treated WTs (Figure 4b). LV cavity volume, reflected in LVEDD, remained unchanged for all cohorts, including AZT-treated TGs (data not shown), suggesting no left ventricular dilation.

\section{Histological Analysis}

H\&E-stained microscopic sections of hearts from TGs and WTs with and without AZT or RCV treatment were analyzed comparatively (original amplification $\times 40$ ). Representative images from each cohort show no significant myocytolytic changes in any of the heart tissues including treatment with AZT (Figure 5a) or RCV (Figure 5b).

\section{DISCUSSION}

TG mice with cardiac-targeted gene expression are useful and powerful tools to define features of cardiac dysfunction, ${ }^{30}$ antiretroviral-associated cardiomyopathy, ${ }^{31}$ genetic changes related to mitochondrial function, ${ }^{32}$ and defects in mitochondrial biogenesis relate to drug toxicity. ${ }^{33}$ We report here a novel in vivo murine model with cardiac-targeted a
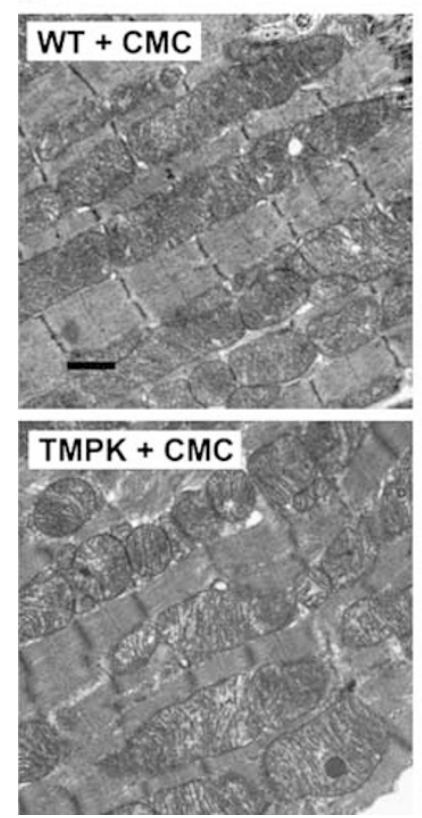
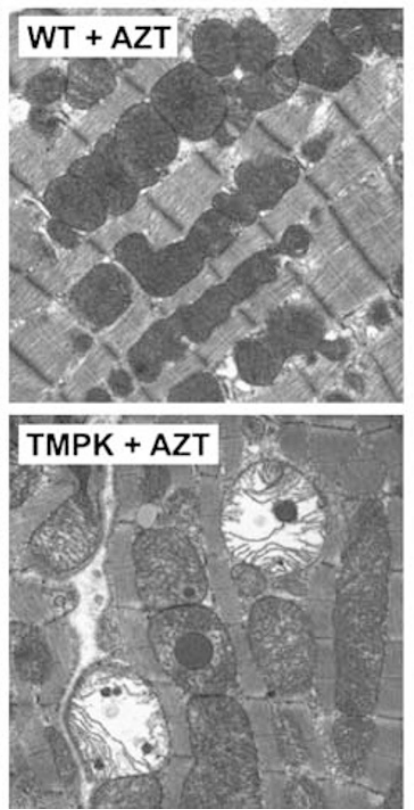

b
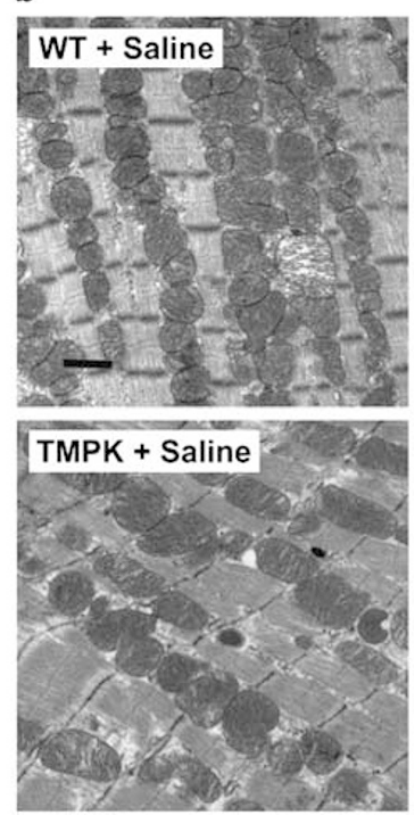
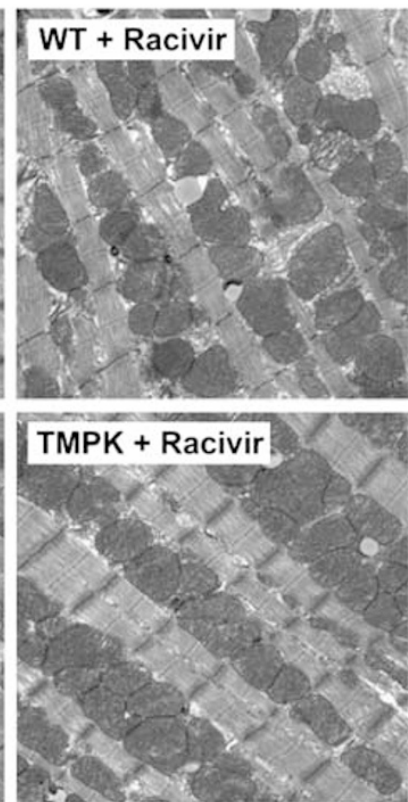

Figure 3 Electron photomicrographs of the mitochondria from cardiac myocytes of TMPK TG and WT treated with AZT or RCV: Representative TEM profiles of cardiac tissues from ' $2 \times 2$ ' studies of TMPK TGs and WTs treated with AZT (a), RCV (b), and their respective vehicle controls are shown. Cardiac myocytes from TMPK TGs treated with AZT show disrupted sarcomeres and tubules with mitochondrial swelling and reduced cristae density (a, lower right panel) compared to vehicle-treated WTs. (b) Changes after treatment with RCV in TGs and WTs were unremarkable. (Original magnification: $\times 22300$, marker indicates $1 \mu \mathrm{m}$.) 
a

TMPK + AZT Monotherapy

LVMass/bw
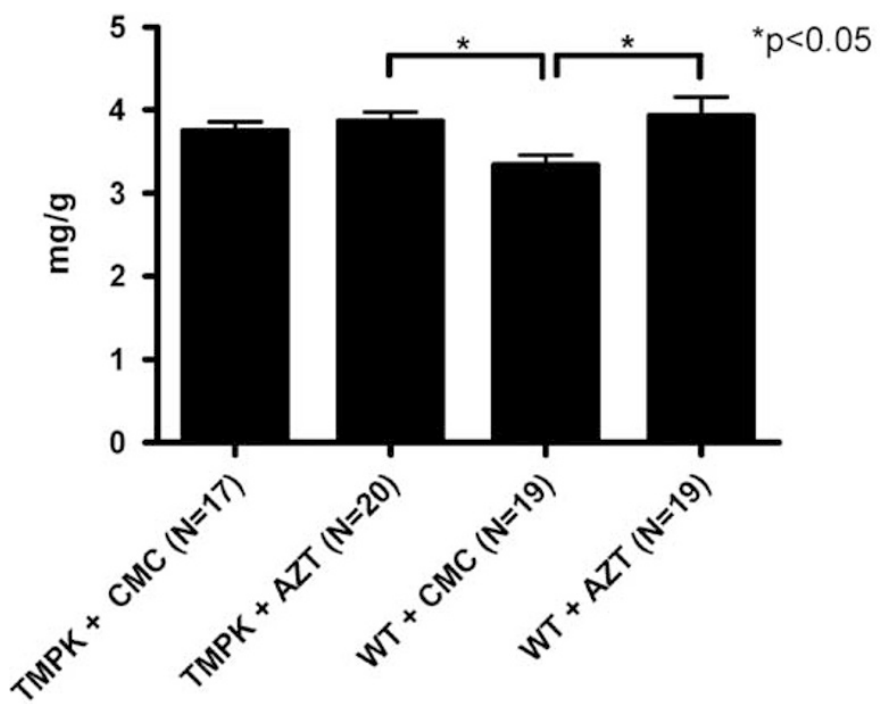

b

TMPK + Racivir Monotherapy

LV Mass/bw

NS

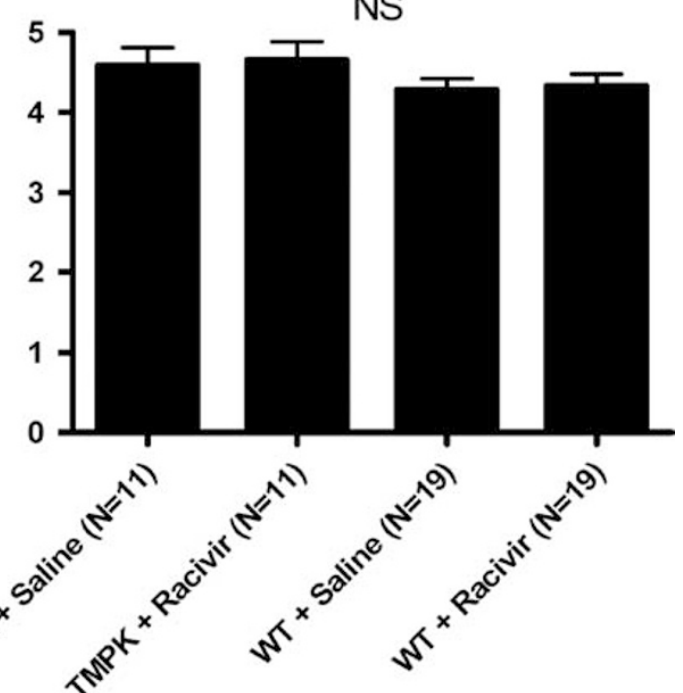

Figure 4 Quantitative analysis of ECHO images. LV mass was determined from ECHO images captured just before termination. Data were normalized to body weight $(\mathrm{mg} / \mathrm{g})$ and plotted as mean \pm s.e.m. (a) AZT treatment increased LV mass in WT and TGs, compared to vehicle-treated WTs $(P<0.05)$. (b) LV mass remained unchanged in TGs and WTs after RCV treatment (right graph).

a

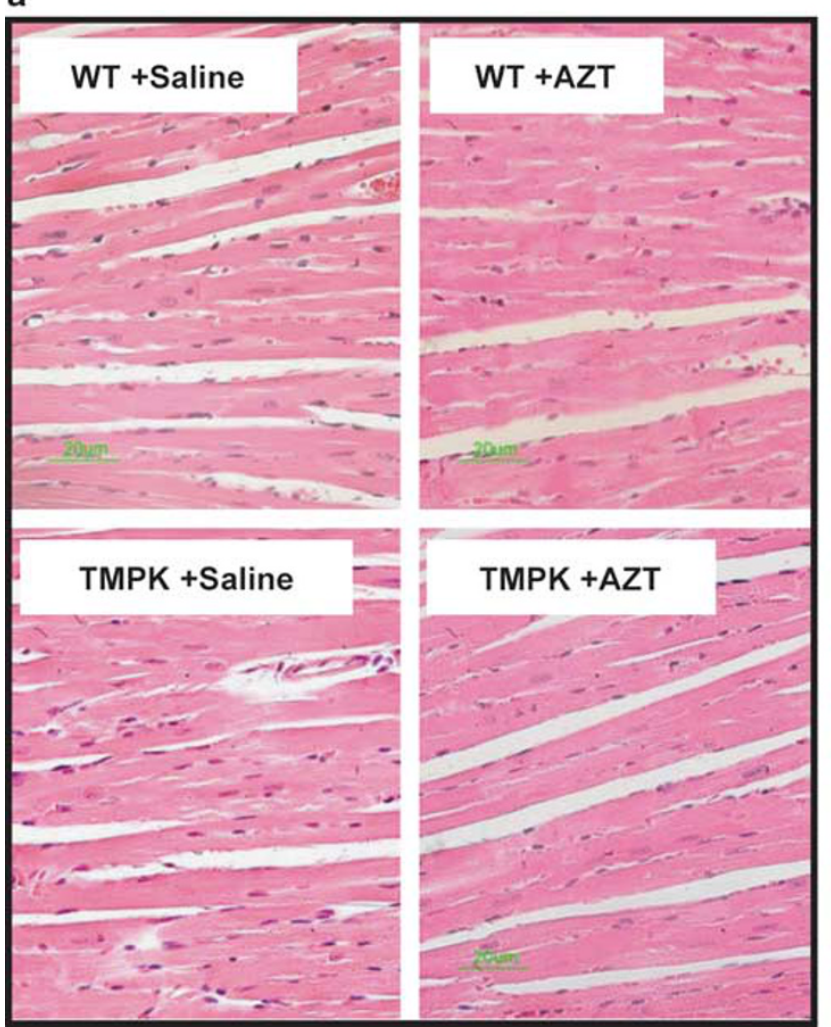

b

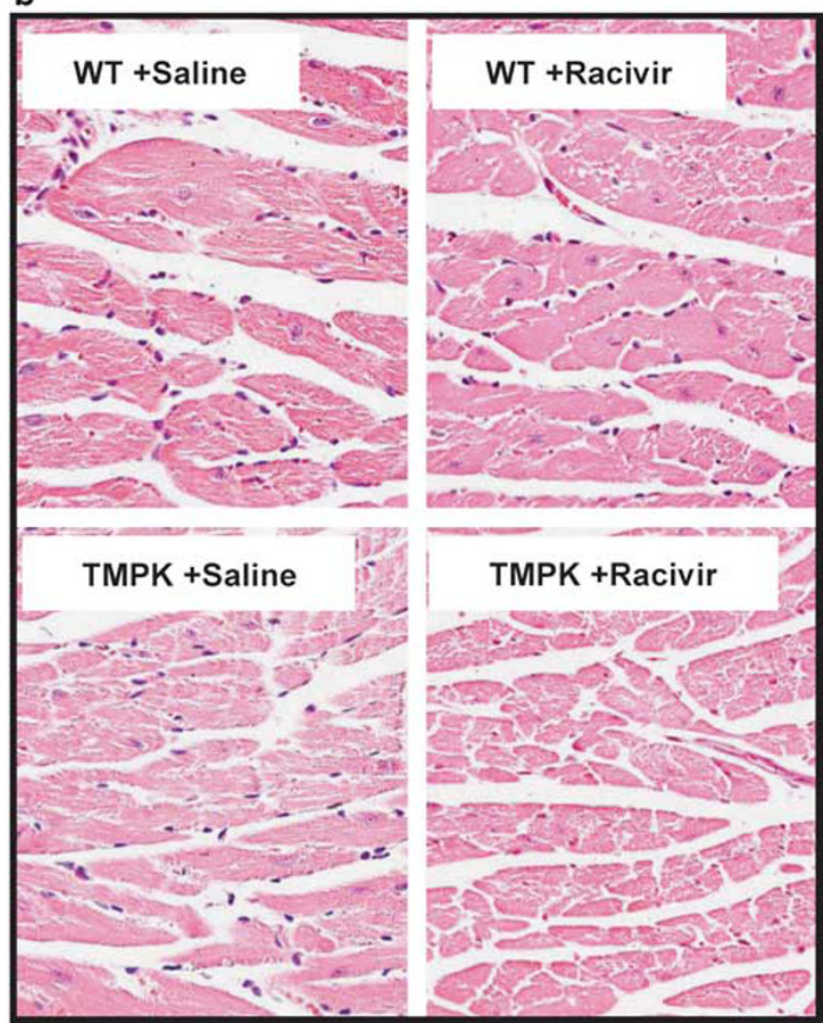

Figure 5 Histological analysis of cardiac tissues from TMPK TGs and WTs treated with AZT or RCV: Parallel H\&E-stained slides were made from gendermatched pairs of cardiac tissues after treatment (35 days). All tissues from TMPK TGs and WTs treated with AZT (a) or RCV (b) showed intact cardiomyocytes with comparable nuclei (original magnification $\times 40$ ). 
overexpression of human TMPK. TMPK is a cell-cycle regulated enzyme, expressed in the $\mathrm{S}$ phase. ${ }^{34}$ Three individual TG lines were generated. Each encoded multiple copies of the human TMPK gene in addition to the native murine TMPK gene. It was imperative to select a screening method that differentiates hTMPK from the native murine TMPK. The successful approach used specific primers to target tandem regions of $\alpha$-MyHC promoter and hTMPK (Figure 1a) and a set of probes that would bind to the amplified product. Our method was reproducible, accurate, and resulted in the amplification and detection only of the hTMPK gene (Figure $\mathrm{lb}$ and $\mathrm{c}$ ). TG line C (possessing the highest gene copy number) was selected because robust overexpression of hTMPK in cardiac muscle was expected to increase phosphorylation of dTMP to dTDP most effectively, and impact mtDNA replication and cardiac function.

TMPK TGs here (vehicle-treated) showed a small yet measurable decrease in mtDNA abundance (Figure 2, $P>0.05$ ). Although overexpression of TMPK may cause increased TDP, dNTP pools are tightly regulated and rely on steady state of a reaction. Increasing the abundance of enzyme alone does not necessarily increase flux through a pathway unless substrate is unlimited. Factors such as availability of substrate (dTMP) demand for product (dTTP), and activities of other enzymes in the phosphorylation and dephosphorylation pathways also have a role.

It could be argued that any potential increase in cardiac mitochondrial dTTP abundance caused by TMPK TGs per se has only moderate impact, as mentioned earlier, through the effects on other nucleoside kinases and on ribonucleotide reductase. Specifically, in vitro studies found dTTP is a feedback inhibitor of human TK $1^{35,36}$ and is a regulator of ribonucleotide reductase. ${ }^{37}$ It remains unclear whether these inhibitory mechanisms can occur in quiescent cells, such as in cardiac myocytes.

In contrast, significant mtDNA depletion, mitochondrial ultrastructural damage, and increased LV mass were found in TMPK TGs treated with AZT. Cardiac overexpression of TMPK, the rate-limiting enzyme in the pathway converting AZT to AZTTP, may have resulted in increased AZTTP levels, which can directly inhibit pol $\gamma^{33}$ However, these experiments offer no direct evidence (ie measurement of dTTP pools) to support this conclusion.

RCV had no impact on TG (or WT) LV mass or mitochondrial ultrastructure. As TMPK kinase is relatively substrate specific for thymidine, the cytidine analog, RCV is not phosphorylated by it. However, RCV treatment of TGs significantly decreased mtDNA abundance (Figure $2 b$ ). This latter finding supports (albeit indirectly) the notion of the absence of a universal mechanism of mitochondrial toxicity of NAs. ${ }^{38}$ Although AZT led to decreased mtDNA abundance and mitochondrial ultrastructural changes that ultimately impacted cardiac function, the effect of RCV was limited to decreased mtDNA abundance alone.
Overexpression of TMPK may also impact dNTP pool balance. A direct assessment of dNTP pools would help define the role of TMPK on mitochondrial dNTPs. Such measurements were not made in these studies. Future studies using TMPK TGs will directly determine dNTP pools.

Overall, initial studies suggest enhanced TMPK activity by genetically engineered overexpression (TG) renders detectable changes in the mtDNA replication with potentially significant subcellular consequences.

\section{ACKNOWLEDGEMENTS}

We thank the Department of Pathology Experimental Core Laboratory for their support. We also thank Sarah Lewis for her assistance. The studies were supported by R01 HL79867 and HL59798 to WL and R01 HL085499 to QY. $\mathrm{JK}$ is a recipient of K01 DK78513.

\section{DISCLOSURE/CONFLICT OF INTEREST}

The authors declare no conflict of interest.

1. Clayton DA. Mitochondrial DNA replication:what we know. IUBMB Life 2003;55:213-217.

2. Bogenhagen D, Clayton DA. Mouse $L$ cell mitochondrial DNA molecules are selected randomly for replication throughout the cell cycle. Cell 1977;11:719-727.

3. Mathews CK. DNA precursor metabolism and genomic stability. FASEB J 2006;20:1300-1314.

4. Mathews CK, Song S. Maintaining precursor pools for mitochondrial DNA replication. FASEB J 2007;21:2294-2303.

5. Nishino I, Spinazzola A, Papadimitriou A, et al. Mitochondrial neurogastrointestinal encephalomyopathy:an autosomal recessive disorder due to thymidine phosphorylase mutations. Ann Neuruo 2000;47:792-800.

6. Ferraro P, Nicolosi L, Bernardi $\mathrm{P}$, et al. Mitochondrial deoxynucleotide pool sizes in mouse liver and evidence for a transport mechanism for thymidine monophosphate. Proc Natl Acad Sci USA 2006;103: 18586-18591.

7. Ferraro $\mathrm{P}$, Bianchi V, Biasin MR, et al. Deoxynucleotide pools and DNA synthesis in resting and PHA-stimulated human lymphocytes treated with mutagens. Exp Cell Res 1992;199:349-354.

8. Eriksson S, Munch-Petersen B, Kierdaszuk B, et al. Expression and substrate specificities of human thymidine kinase 1, thymidine kinase 2 and deoxycytidine kinase. Adv Exp Med Biol 1991;309B:239-243.

9. Hosseini $\mathrm{SH}$, Kohler JJ, Haase $\mathrm{CP}$, et al. Targeted transgenic overexpression of mitochondrial thymidine kinase (TK2) alters mitochondrial DNA (mtDNA;) and mitochondrial polypeptide abundance: transgenic TK2, mtDNA, and antiretrovirals. Am J Pathol 2007;170:865-874.

10. Kohler JJ, Hosseini SH, Cucoranu I, et al. Murine cardiac mtDNA:effects of transgenic manipulation of nucleoside phosphorylation. Lab Invest 2009:89:122-130.

11. Van Rompay AR, Johansson M, Karlsson A. Phosphorylation of nucleosides and nucleoside analogs by mammalian nucleoside monophosphate kinases. Pharmacol Ther 2000;87:189-198.

12. Palmieri F. The mitochondrial transporter family (SLC25):physiological and pathological implications. Pflugers Arch 2004;447:689-709.

13. Galmarini CM, Mackey JR, Dumontet C. Nucleoside analogues and nucleobases in cancer treatment. Lancet Oncol 2002;3:415-424.

14. Lavie A, Schlichting I, Vetter IR, et al. The bottleneck in AZT activation. Nat Med 1997;3:922-924.

15. Lavie A, Konrad M. Structural requirements for efficient phosphorylation of nucleotide analogs by human thymidylate kinase. Mini Rev Med Chem 2004;4:351-359.

16. Robbins J, Palermo J, Rindt H. In vivo definition of a cardiac specific promoter and its potential utility in remodeling the heart. Ann N Y Acad Sci 1995;752:492-505.

17. Lewis W, Kohler JJ, Hosseini SH, et al. Antiretroviral nucleosides, deoxynucleotide carrier and mitochondrial DNA:evidence supporting the DNA pol gamma hypothesis. Aids 2006;20:675-684. 
18. Brundiers $R$, Lavie $A$, Veit $T$, et al. Modifying human thymidylate kinase to potentiate azidothymidine activation. J Biol Chem 1999;274:35289-35292.

19. Ostermann N, Schlichting I, Brundiers R, et al. Insights into the phosphoryltransfer mechanism of human thymidylate kinase gained from crystal structures of enzyme complexes along the reaction coordinate. Struct Fold Des 2000;8:629-642.

20. Hoit BD, Walsh RA. Cardiovascular Physiology in the Genetically Engineered Mouse (2nd edition), Kluwer Academic: Norwell, 2002 pp 403.

21. Lewis W, Grupp IL, Grupp G, et al. Cardiac dysfunction occurs in the HIV-1 transgenic mouse treated with zidovudine. Lab Invest 2000;80:187-197.

22. Lewis $\mathrm{W}$, Haase CP, Miller YK, et al. Transgenic expression of the deoxynucleotide carrier causes mitochondrial damage that is enhanced by NRTIs for AIDS. Lab Invest 2005;85:972-981.

23. Kohler JJ, Hosseini SH, Green E, et al. Cardiac-targeted transgenic mutant mitochondrial enzymes:mtDNA defects, antiretroviral toxicity and cardiomyopathy. Cardiovasc Toxicol 2008;8:57-69.

24. Cote HC, Yip B, Asselin JJ, et al. Mitochondrial:nuclear DNA ratios in peripheral blood cells from human immunodeficiency virus (HIV)infected patients who received selected HIV antiretroviral drug regimens. J Infect Dis 2003;187:1972-1976.

25. Lewis W, Day BJ, Kohler JJ, et al. Decreased mtDNA, oxidative stress, cardiomyopathy, and death from transgenic cardiac targeted human mutant polymerase gamma. Lab Invest 2007;87:326-335.

26. Lewis W, Haase CP, Raidel SM, et al. Combined antiretroviral therapy causes cardiomyopathy and elevates plasma lactate in transgenic AIDS mice. Lab Invest 2001;81:1527-1536.

27. Dalakas MC, Illa I, Pezeshkpour GH, et al. Mitochondrial myopathy caused by long-term zidovudine therapy. N Engl J Med 1990;322:1098-1105.
28. Kohler JJ, Cucoranu I, Fields E, et al. Transgenic mitochondrial superoxide dismutase and mitochondrially targeted catalase prevent antiretroviral-induced oxidative stress and cardiomyopathy. Lab Invest 2009;89:782-790.

29. Balzarini J, Pauwels $\mathrm{R}, \mathrm{Baba} \mathrm{M}$, et al. The in vitro and in vivo antiretrovirus activity, and intracellular metabolism of $3^{\prime}$-azido- $2^{\prime}, 3^{\prime}$ dideoxythymidine and $2^{\prime}, 3^{\prime}$-dideoxycytidine are highly dependent on the cell species. Biochem Pharmacol 1988;37:897-903.

30. Robbins J. Remodeling the cardiac sarcomere using transgenesis. Annu Rev Physiol 2000;62:261-287.

31. Lewis W. Defective mitochondrial DNA replication and NRTIs:pathophysiological implications in AIDS cardiomyopathy. Am J Physiol Heart Circ Physiol 2003;284:H1-H9.

32. Russell LK, Finck BN, Kelly DP. Mouse models of mitochondrial dysfunction and heart failure. J Mol Cell Cardiol 2005;38:81-91.

33. Lewis W, Day BJ, Copeland WC. Mitochondrial toxicity of nrti antiviral drugs:an integrated cellular perspective. Nat Rev Drug Discov 2003;2:812-822.

34. Huang SH, Tang A, Drisco B, et al. Human dTMP kinase:gene expression and enzymatic activity coinciding with cell cycle progression and cell growth. DNA Cell Biol 1994;13:461-471.

35. Bresnick E, Thompson UB, Morris HP, et al. Inhibition of thymidine kinase activity in liver and hepatomas by TTP and d-CTP. Biochem Biophys Res Commun 1964;16:278-284.

36. Barrie SE, Paine RM, Stock JA, et al. Enzyme inhibition by phosphonate analogues of dTTP. Adv Exp Med Biol 1984;165:371-374.

37. $\mathrm{Xu} \mathrm{H}$, Faber $\mathrm{C}$, Uchiki $\mathrm{T}$, et al. Structures of eukaryotic ribonucleotide reductase I provide insights into dNTP regulation. Proc Natl Acad Sci USA 2006;103:4022-4027.

38. Lund KC, Peterson LL, Wallace KB. Absence of a universal mechanism of mitochondrial toxicity by nucleoside analogs. Antimicrob Agents Chemother 2007;51:2531-2539. 